Revue internationale P.M.E.

Économie et gestion de la petite et moyenne entreprise

Revure

internationale

PME

\title{
La veille stratégique chez les PME de haute technologie: une étude de cas par comparaisons intersites
}

\section{Josée Audet}

Volume 16, numéro 2, 2003

URI : https://id.erudit.org/iderudit/1008441ar

DOI : https://doi.org/10.7202/1008441ar

Aller au sommaire du numéro

Éditeur(s)

Presses de l’Université du Québec

ISSN

0776-5436 (imprimé)

1918-9699 (numérique)

Découvrir la revue

Citer cette note

Audet, J. (2003). La veille stratégique chez les PME de haute technologie: une étude de cas par comparaisons intersites. Revue internationale P.M.E., 16(2), 105-129. https://doi.org/10.7202/1008441ar
Résumé de l'article

L’objectif de cette étude était de relever des caractéristiques de veille stratégique susceptibles d'être associées à la performance des PME œuvrant en haute technologie. La stratégie de recherche favorisée pour atteindre cet objectif a été l'étude de cas par comparaisons intersites. Les résultats ont permis d'ébaucher un profil des activités de veille caractérisant les PME de haute technologie connaissant du succès. La propension à l'innovation des membres de l'équipe dirigeante semble être le catalyseur des activités de veille, stimulant et orientant les efforts de veille. Ainsi, la veille est motivée par la recherche d'occasions d'affaires et elle vise principalement l'horizon à long terme. Par ailleurs, les dirigeants gèrent de façon stratégique leurs réseaux d'information, afin d'y puiser les informations nécessaires à l'innovation. 


\title{
Note de recherche \\ La veille stratégique chez les PME de haute technologie: une étude de cas par comparaisons intersites
}

Josée AUDET

Université du Québec à Trois-Rivières

\author{
MOTS CLÉS \\ Stratégie - Veille stratégique - Veille technologique \\ Vigie - Innovation
}

\begin{abstract}
RÉSUMÉ
L'objectif de cette étude était de relever des caractéristiques de veille stratégique susceptibles d'être associées à la performance des PME œuvrant en haute technologie. La stratégie de recherche favorisée pour atteindre cet objectif a été l'étude de cas par comparaisons intersites. Les résultats ont permis d'ébaucher un profil des activités de veille caractérisant les PME de haute technologie connaissant du succès. La propension à l'innovation des membres de l'équipe dirigeante semble être le catalyseur des activités de veille, stimulant et orientant les efforts de veille. Ainsi, la veille est motivée par la recherche d'occasions d'affaires et elle vise principalement l'horizon à long terme. Par ailleurs, les dirigeants gèrent de façon stratégique leurs réseaux d'information, afin d'y puiser les informations nécessaires à l'innovation.
\end{abstract}

\section{L'AUTEURE}

JoSÉE AUDET a fait ses études doctorales à l'Université Laval, sa thèse portant sur la veille stratégique chez les PME. Elle est professeure à l'Université du Québec à Trois-Rivières et membre de l'Institut de recherche sur les PME. Ses activités de recherche visent à la fois la gestion stratégique des PME et le processus de création d'entreprise. Adresse: Université du Québec à Trois-Rivières, Département des sciences de la gestion, C.P. 500, Trois-Rivières, Québec, Canada, G9A 5H7. Téléphone : (819) 376-5011 poste 3146. Courriel : <josee_audet@uqtr.ca>. 


\begin{abstract}
The scope of this study was to identify strategic scanning characteristics that were likely to contribute to the success of high technology SMEs. A multi-site case study methodology was used to achieve this research objective. A profile of strategic scanning characteristics likely to lead to higher performance among high technology SMEs emerged from our research in the field. The innovation orientation of the top management team is the driving force behind scanning activities, encouraging and guiding these activities. Scanning is thus opportunity driven and geared towards a long term horizon. Results also suggest that information networks are strategically managed to yield information that will contribute to innovation.
\end{abstract}

\title{
RESUMEN
}

El objetivo del presente estudio consiste en identificar las características de la observación estratégica susceptibles de estar asociadas con las prestaciones de las PyMEs que obran en alta tecnología. La estrategia de investigación empleada para lograr ese objetivo ha sido el estudio de caso por comparación inter-sedes. Los resultados han permitido dibujar un perfil de las actividades de observación que caracterizan a las PyMEs de alta tecnología que tienen éxito. La propensión a la innovación de los miembros del grupo dirigente parece ser el catalizador de las actividades de observación, que quedan así estimuladas y orientadas. La observación es motivada por la búsqueda de oportunidades y está dirigida principalmente hacia una perspectiva a largo plazo. Por otro lado, los dirigentes administran sus redes de información de manera estratégica, con el fin de obtener informaciones necesarias para la innovación.

\section{ZUSAMMENFASSUNG}

Die Zielsetzung dieser Studie bestand darin, Eigenschaften von strategischen Überwachungsmechanismen zu identifizieren, die mit der Leistung von hoch technologisierten KMU verbunden werden können. Um diese Zielsetzung zu erreichen war die bevorzugte Forschungsstrategie Fallstudienvergleiche zwischen verschiedenen Standorten durchzuführen. Die Ergebnisse haben erlaubt, ein Profil der Überwachungsmechanismen zu skizzieren, die den Erfolg von KMU mit hoher Technologie charakterisieren. Die Neigung zur Innovation der Mitglieder des leitenden Teams scheint der Katalysator und Anreiz für strategische Überwachungsaktivitäten zu sein. Somit werden die Überwachungsmechanismen durch gelegentliche, intuitive Nachforschungen motiviert und langfristig ausgerichtet. Andererseits verwalten die Führungskräfte strategisch ihre Informationsnetze in solcher Art und Weise, dass die für die Innovation notwendigen Informationen ausgeschöpft werden.

\section{Introduction}

Au cours des dernières années, nous avons été témoins d'une profonde transformation de l'économie mondiale, caractérisée par un fort niveau de concurrence alimenté par la globalisation des marchés et le rythme effréné auquel se succèdent 
les innovations technologiques. Ce contexte fait en sorte qu'il est difficile pour une PME d'être à la fine pointe de la technologie et, surtout, d'y demeurer. Les compétiteurs sont féroces ; souvent, ils ont accès à des ressources financières considérables et, qui plus est, fusent des quatre coins de la planète. Le succès à long terme d'une PME technologique requiert plus qu'un positionnement adéquat dans son marché actuel : elle doit de surcroît imaginer celui de demain (Hamel et Prahalad, 1994). Il ne s'agit pas exactement de prévoir l'avenir tel un devin mais plutôt de s'outiller prospectivement pour réagir et évoluer en même temps que le système (Courville, 1994). Pour y parvenir, l'entreprise doit avoir en main les informations qui lui permettront justement de se préparer adéquatement. C'est par le biais des activités de veille stratégique que les informations provenant de l'environnement externe de l'entreprise seront recueillies, partagées et interprétées, pour ultimement alimenter le processus de réflexion stratégique des dirigeants.

Par leurs fonctions, les activités de veille stratégique semblent susceptibles d'avoir un impact sur la performance de l'entreprise puisqu'elles permettent d'obtenir les informations essentielles à son adaptation. Or, malgré ce rôle crucial qu'est appelée à jouer la veille stratégique, trop peu de recherches ont porté sur la relation susceptible d'exister entre la veille et la performance de l'entreprise. Cela constitue un problème de taille pour le dirigeant de PME technologique soucieux d'adopter des pratiques de veille de nature à lui procurer des informations utiles à sa réflexion stratégique et, ultimement, à contribuer à l'essor de son entreprise. Lesca souligne d'ailleurs à juste titre que « les entreprises désireuses d'implanter la veille stratégique se heurtent à l'absence de modèles, de méthodes et d'outils susceptibles de les aider » (1994, p. 36). Ce manque de connaissances risque d'avoir des effets encore plus néfastes chez les PME de haute technologie puisque leur environnement d'affaires est parmi les plus complexes et les plus turbulents, d'où un plus grand besoin d'informations. Or, par leur dynamisme et leur créativité, ces entreprises sont appelées à jouer un rôle crucial au sein de la nouvelle économie. La question de recherche suivante s'impose donc: "Quelles caractéristiques des activités de veille stratégique sont susceptibles d'être associées au succès des PME québécoises œuvrant en haute technologie ?»

Dans un premier temps, un survol des écrits relatifs à la veille stratégique sera présenté afin de bien situer la problématique de recherche. Le cadre théorique qui a guidé la démarche de recherche sera ensuite proposé, suivi d'une description de la méthodologie de recherche préconisée, soit l'étude de cas par comparaisons intersites. Les résultats seront présentés et commentés, d'abord ceux qui sont communs à l'ensemble des PME de haute technologie observées, puis ceux qui se rapportent aux PME les plus performantes de l'échantillon.

Revue internationale P.M.E., vol. 16, $\mathrm{n}^{\circ}$ 2, 2003

(C) 2003 - Presses de l'Université du Québec 


\section{Recension des écrits}

Qu'entend-on par veille stratégique? Selon Aguilar, pionnier de la recherche en matière de veille stratégique, la veille stratégique consiste à saisir les informations «relatives aux événements et aux relations prenant place dans l'environnement externe de l'entreprise, informations dont la connaissance est susceptible d'aider l'équipe dirigeante dans ses choix stratégiques quant au futur de l'entreprise » (1967, p. 1). Pearce II, Chapman et David (1982) comparent la planification stratégique au gouvernail qui tient l'entreprise sur le cap alors que la veille stratégique serait le radar informant le pilote des conditions de navigation qu'il risque de trouver sur sa route. Dess et Miller considèrent, pour leur part, que la veille stratégique ne se limite pas à la seule collecte d'informations ; selon eux, elle serait un «processus incluant à la fois l'écoute de l'environnement externe et la saisie d'informations, ce processus se continuant par l'évaluation de l'information recueillie et ultimement sa transmission aux personnes clés de l'organisation » (1993, p. 38). Dans le même ordre d'idées, Lesca et Caron font référence à « un processus informationnel par lequel l'entreprise détecte et traite les signaux annonciateurs d'événements susceptibles d'influencer sa pérennité » (1995, p. 58). Cette vision plus englobante du concept de veille stratégique a été favorisée dans le cadre de la présente étude. La veille stratégique correspond ainsi au processus par lequel les membres d'une organisation amassent, partagent et donnent un sens à l'information relative à l'environnement externe de la firme ; cet exercice ayant pour but d'alimenter le processus de réflexion stratégique des dirigeants de l'entreprise. À noter que quatre types de veille sont répertoriés dans les écrits : la veille technologique, la veille concurrentielle, la veille commerciale et la veille sociétale (Martinet et Ribault, 1989), la veille stratégique étant, quant à elle, un concept plus large regroupant ces quatre types particuliers de veille (Pateyron, 1998).

La recension des écrits portant sur la veille stratégique révèle l'existence d'une majorité de travaux empiriques de nature plutôt descriptive. Ils nous renseignent notamment sur les secteurs de l'environnement que les dirigeants scrutent, les moyens qu'ils privilégient pour se renseigner ou la fréquence à laquelle ils s'adonnent à des activités de veille (Aguilar, 1967; Johnson et Kuehn, 1987 ; Smeltzer, Fann et Nikolaisen, 1988 ; Auster et Choo, 1994 ; Pineda et al., 1998). Certains auteurs ont plutôt tenté de développer des typologies de systèmes de veille, ces systèmes se distinguant selon le degré d'évolution des pratiques de veille de l'entreprise (Fahey et King, 1977 ; Jain, 1984 ; Julien et al., 1999). D'autres auteurs ont cherché à mettre au jour une association entre la veille stratégique et la stratégie (Hambrick, 1982 ; Jennings et Lumpkin, 1992 ; Hagen et Amin, 1995 ; Beal, 2000), l'incertitude stratégique de l'environnement (Daft, Sormunen et Parks, 1988 ; Boyd, 1989 ; Choo, 1993 ; Sawyerr, 1993), ou les caractéristiques personnelles du dirigeant (Hambrick, 1981 ; Kaish et Gilad, 1991 ; Cooper, Folta et Woo, 1995).

Revue internationale P.M.E., vol. 16, nº 2, 2003

(C) 2003 - Presses de l'Université du Québec 
Des écrits font aussi état de l'importance que revêt pour le dirigeant de PME son réseau de contacts, notamment afin d'y puiser des informations (Dubini et Aldrich, 1991 ; Oughebbi, 1993 ; Donckels et Lambrecht, 1997). Certains auteurs sont même d'avis qu'il existerait un lien entre l'intensité des activités de réseautage du dirigeant de PME et la croissance de son entreprise (Hansen, 1995 ; Ostgaard et Birley, 1996). Ce lien serait vraisemblablement encore plus fort dans un contexte de grande volatilité et de haute incertitude, comme celui dans lequel évoluent les PME de haute technologie (Sawyerr et McGee, 1999). La richesse de l'information susceptible d'émaner du réseau de contacts de l'équipe dirigeante contribuerait, d'une part, à accélérer le processus d'innovation et, d'autre part, à réduire l'incertitude se dégageant de l'environnement, ce qui en retour faciliteraient la prise de décision et le passage à l'action (Julien, 1996).

Par ailleurs, bien que de prime abord les activités de veille soient de nature à avoir un impact sur la performance de l'entreprise, très peu d'auteurs se sont penchés sur la relation existant entre les activités de veille et cette performance. Parmi eux, Dollinger (1984) a vérifié auprès de 82 dirigeants de PME s'il y avait une relation entre l'intensité des activités de veille (boundary spanning activities), l'habileté du dirigeant à traiter l'information (information processing capability) et la performance de l'entreprise. Le premier construit a été mesuré à partir du rapport entre le nombre d'heures passées par le dirigeant en compagnie de personnes susceptibles de lui transmettre des informations stratégiques et le nombre d'heures travaillées dans la semaine, en tenant aussi compte du nombre de contacts hebdomadaires avec ces informateurs. Le construit de l'habileté du dirigeant à traiter l'information a, quant à lui, été mesuré en fonction du degré d'intolérance à l'ambiguïté du dirigeant et de son niveau de complexité intégrative. Les résultats démontrent que la première mesure du construit de l'intensité des activités de veille explique de manière significative la variance de la performance, tandis que, pour la seconde, l'association positive est non significative. Par ailleurs, la relation entre l'habileté du dirigeant à traiter l'information et la performance n'a pu être établie de façon convaincante.

Les recherches de Daft, Sormunen et Parks (1988) effectuées auprès de 50 firmes de taille moyenne à grande (ventes de 2 à 500 millions de dollars) avaient pour but de vérifier l'existence d'une relation entre l'intensité de la veille pour un secteur de l'environnement donné et l'incertitude stratégique perçue pour ce secteur et, le cas échéant, d'établir si cette relation variait selon le niveau de performance de l'entreprise. Dans cette étude, l'environnement de la firme comprend à la fois l'environnement immédiat (clients, concurrents et technologie) et l'environnement général (secteurs économique, socioculturel et réglementaire). Quant au concept d'incertitude stratégique, il renvoie à une mesure combinée de l'importance stratégique perçue d'un secteur de l'environnement et de son degré de complexité et de turbulence. Premier constat: l'association entre l'intensité relative de la veille 
et le niveau d'incertitude stratégique d'un secteur est plus forte chez les firmes les plus performantes. En d'autres termes, les dirigeants des entreprises plus performantes modifient l'intensité de leur veille en fonction du niveau d'incertitude stratégique qu'ils perçoivent pour chacun des secteurs de l'environnement. Deuxième constat : le champ de vision de ces dirigeants est plus large et, de façon générale, l'intensité de leur veille, plus élevée.

Kaish et Gilad (1991) ont, pour leur part, mis en évidence que les activités de veille stratégique perdent de leur importance au fur et à mesure que les PME gagnent en expérience et en profitabilité. Les auteurs tentent d'expliquer ce phénomène pour le moins étonnant en posant l'hypothèse que les propriétairesdirigeants, après avoir atteint un certain rythme de croisière, concentreraient leur énergie sur la gestion interne plutôt que sur la recherche de nouvelles occasions d'affaires.

Finalement, dans le cadre d'une étude menée auprès de 331 directeurs d'hôpitaux, Subramanian, Kumar et Yauger (1994) ont vérifié l'existence de différences de performance parmi les entreprises selon l'évolution de leur système de veille. Les deux dimensions de veille retenues pour caractériser les systèmes de veille sont l'intensité de la veille relativement à différents secteurs de l'environnement immédiat et le degré d'intégration de l'information recueillie au processus de décision stratégique. Quatre types de système de veille ont été mis en lumière, allant du plus primitif au plus évolué. Il appert que les mesures de performance des hôpitaux dont le système de veille est considéré comme plus évolué sont nettement plus élevées que celles des autres hôpitaux.

En rétrospective, s'il faut se fier aux résultats des études empiriques recensées, les caractéristiques de veille ayant un impact significatif sur la performance de l'entreprise sont l'intensité générale des activités de veille, le degré d'intégration de l'information recueillie au processus stratégique et, finalement, le degré d'adéquation entre l'intensité de la veille pour un secteur donné de l'environnement et l'incertitude stratégique perçue pour ce secteur. Il importe par ailleurs de souligner qu'à l'exception des recherches de Kaish et Gilad (1991) et de celles de Dollinger (1984), les études portant sur la relation entre la veille et la performance ont été menées auprès de grandes entreprises. Or, les deux recherches ayant pour objet la veille chez les PME ne traitaient que de deux aspects des activités de veille, soit leur intensité et les habiletés du veilleur à traiter l'information, et les PME observées n'étaient pas des entreprises œuvrant en haute technologie. De surcroît, les résultats obtenus par Kaish et Gilad relativement à l'intensité de la veille contredisent carrément ceux de Dollinger et les résultats de ce dernier quant aux habiletés du veilleur à traiter l'information ne sont pas probants. C'est donc dire qu'il n'y a pas de bases théoriques solides et reconnues pouvant expliquer la relation entre la veille et la performance dans le contexte particulier des PME de haute technologie. 


\section{Cadre conceptuel préliminaire}

À défaut d'avoir pu relever dans les écrits des caractéristiques de la veille qui soient associées à la performance des PME de haute technologie, il a été décidé de retenir les trois dimensions de la veille associées de façon significative à la performance des entreprises sans distinction quant à la taille de ces dernières ou leur secteur d'activité. Ces dimensions sont l'intensité de la veille, l'intégration de l'information au processus stratégique et l'adéquation entre l'intensité de la veille et l'incertitude stratégique perçue des secteurs scrutés (voir figure 1). Ce choix en apparence réducteur ne l'est pas en réalité. En effet, comme nous l'expliquerons dans la prochaine section, la méthodologie de recherche privilégiée laisse la flexibilité requise pour aller au-delà des limites posées par le cadre conceptuel. C'est d'ailleurs la raison pour laquelle ce cadre est qualifié de «préliminaire».

FIGURE 1

\section{Cadre conceptuel préliminaire des activités de veille de la PME de haute technologie}

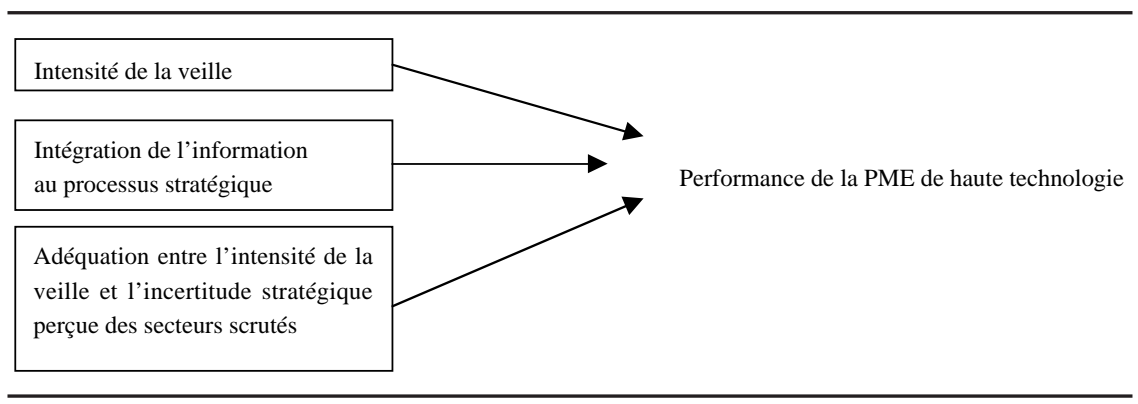

Par ailleurs, il est raisonnable de croire qu'il existe d'autres caractéristiques de la veille stratégique de nature à avoir un impact sur la performance des PME de haute technologie, notamment celles sélectionnées par différents auteurs pour créer des typologies de système de veille (Fahey et King, 1977; Jain, 1984 ; Julien et al., 1999). Comme nous l'avons mentionné, ces typologies distinguent le niveau d'évolution des systèmes de veille selon plusieurs dimensions. Or, bien que la relation entre le niveau d'évolution de ce système et la performance de l'entreprise n'ait jamais été vérifiée, il est vraisemblable que plus un système de veille est évolué, plus il est susceptible de contribuer à la réussite de la firme. Les dimensions les plus susceptibles d'avoir un impact sur la performance de PME de haute technologie ont donc été établies et ces dimensions ont été qualifiées de «variables exploratoires ». Bien qu' elles ne fassent pas partie du cadre conceptuel initial à proprement parler, elles ont été opérationnalisées et mesurées lors de la collecte de données, et ce afin d'avoir en main toutes les informations nécessaires pour analyser en profondeur ces données si toutefois elles se révélaient significatives. Les variables 
exploratoires ainsi retenues sont les suivantes : le degré de structuration des efforts de veille, l'horizon temporel de la veille, la motivation sous-tendant l'effort de veille, la place qu'occupe la veille au sein de la culture organisationnelle et l'appartenance du dirigeant à un réseau d'information source d'informations riches.

\section{Méthodologie de recherche}

\subsection{L'étude de cas par comparaisons intersites}

La recension des écrits n'ayant pas mis en évidence l'existence de solides bases théoriques pouvant soutenir une recherche menée selon l'approche hypothéticodéductive, il a été décidé d'adopter plutôt une approche flexible et ouverte, permettant d'explorer le phénomène en profondeur. L'approche méthodologique retenue est l'étude de cas par comparaisons intersites. Cette stratégie de recherche a été développée par d'Amboise (1996), qui s'est largement inspiré des travaux de Yin (1984, 1993, 1994) et d'Eisenhardt (1989).

L'étude de cas par comparaisons intersites se veut une démarche à la fois déductive et inductive. Elle est déductive en ce qu'elle requiert du chercheur qu'il détermine les paramètres de la recherche avant de commencer son travail sur le terrain (question de recherche, recension des écrits, cadre conceptuel et devis de recherche). À ce niveau, elle s'apparente à l'approche hypothéticodéductive classique. De façon concrète, cela signifie que toutes les variables à être observées sont opérationnalisées, cela incluant le recours à des indicateurs et des mesures. À noter que ces mesures ne servent nullement à des fins d'analyse statistique, elles servent plutôt à faire ressortir les caractéristiques du phénomène observé qui semblent les plus significatives. Elles attirent l'attention du chercheur qui complémente alors son interprétation des mesures à l'aide des données qualitatives recueillies. Les comparaisons entre cas s'en trouvent également facilitées puisque les différences se repèrent aisément lorsqu'elles sont quantifiées.

L'étude de cas par comparaisons intersites est aussi inductive en ce qu'elle accorde une très grande ouverture au chercheur face aux phénomènes à être appréhendés sur le terrain. Plus précisément, ce dernier a la latitude de recueillir toute donnée susceptible de l'aider à mieux comprendre le phénomène qu'il observe, les variables du cadre conceptuel initial ne servant que de point de départ à la démarche de recherche. Cette ouverture a justifié la décision de trouver des variables exploratoires concurremment à l'élaboration du cadre conceptuel. De plus, le guide d'entrevue a été élaboré de façon à aller bien au-delà de simples mesures de variables présélectionnées, des questions ouvertes invitant les répondants à communiquer toute information relative à la veille qui pourrait leur venir à l'esprit. Ceux-ci ont donc eu l'occasion d'exprimer librement leurs pensées à maintes reprises au cours de l'entretien. 
Par ailleurs, contrairement à l'approche classique qui préconise un choix aléatoire de l'échantillon afin de permettre une généralisation des résultats, les cas sont plutôt choisis selon le principe de l'échantillonnage délibéré (Merriam, 1998). Les cas sont sélectionnés selon la richesse de l'information que le chercheur estime être en mesure d'y recueillir, tout en tenant compte de dimensions théoriques déterminées au préalable pour baliser la sélection. Ces dimensions sont habituellement des variables reconnues dans les écrits comme étant susceptibles d'avoir un impact sur le phénomène observé. Il est préférable de choisir des cas extrêmes selon les dimensions théoriques retenues, et ce afin de mettre en évidence les différences et les similitudes entre les cas, cela facilitant d'autant l'analyse subséquente des données.

L'analyse des données n'est pas basée sur des tests statistiques mais plutôt sur des techniques de nature qualitative. La première étape consiste à effectuer une étude en profondeur de chacun des cas observés ; il s'agit de l'analyse intrasite. Cela vise notamment à mettre en relief les observations qui semblent les plus significatives par rapport au phénomène étudié. Ce travail permet aussi de réduire le volume d'information avec lequel le chercheur doit jongler et de classer les données retenues selon une certaine logique. Dans le cas présent, ce premier travail d'analyse a résulté en la rédaction d'un profil d'une vingtaine de pages pour chacune des entreprises étudiées.

Une fois cette étape d'analyse préliminaire franchie, la technique principalement utilisée est celle de la recherche de constantes d'un cas à l'autre (crosscase search for patterns), c'est-à-dire la recherche de constantes en comparant de manière systématique et itérative les cas entre eux (Eisenhardt, 1989). L'objectif visé est de dégager un ensemble de caractéristiques et de relations qui soient uniques à un groupe ou sous-groupe de cas. C'est de ces multiples confrontations qu'émergent graduellement des constantes qui servent d'ébauches à une théorie qui va au-delà du cas particulier. Comme le soulignent Huberman et Miles, «la comparaison entre sites ou cas permet d'établir le degré de généralité d'un résultat ou d'une explication et simultanément d'identifier les conditions dans lesquelles ce résultat va apparaître (1991, p. 272). Éventuellement, la configuration obtenue est confrontée au cadre conceptuel préliminaire afin de vérifier son bien-fondé. Au besoin, le cadre préliminaire est ajusté de manière à ce qu'il colle plus étroitement à la réalité ayant émergé du terrain. Cette évolution du cadre conceptuel préliminaire est non seulement prévisible mais aussi souhaitable puisque le but ultimement recherché est d'arriver à un modèle théorique qui décrive le plus fidèlement possible le phénomène tel qu'il a été appréhendé sur le terrain. En d'autres termes, le cadre conceptuel émergent exprime directement la réalité observée sur le terrain alors que le cadre conceptuel préliminaire, lui, est le reflet des connaissances théoriques et empiriques recensées.

Revue internationale P.M.E., vol. 16, $\mathrm{n}^{\circ}$ 2, 2003

(C) 2003 - Presses de l'Université du Québec 
Une technique complémentaire d'analyse est aussi privilégiée, soit l'élaboration d'explications (Yin, 1994). Cette technique d'analyse consiste à expliquer un phénomène en construisant cas par cas, de manière graduelle et itérative, un ensemble de liens de causalité décrivant le phénomène en question. Pour élaborer un tel ensemble de liens logiques, le chercheur s'inspire à la fois des écrits théoriques recensés et de sa compréhension du phénomène tel qu'il s'exprime dans le cadre conceptuel émergent. L'idée derrière cet exercice est de dégager une explication qui soit justifiable sur un plan théorique tout en étant solidement ancrée dans la réalité observée. Dans la mesure où l'explication avancée se trouve vérifiée pour chacun des sites, la confiance pouvant être accordée aux résultats exprimés sous la forme du cadre conceptuel émergent en est d'autant grandie.

\subsection{Les mesures des variables retenues}

Comme plusieurs des variables du cadre conceptuel se retrouvaient aussi dans les travaux de Daft, Sormunen et Parks (1988), l'intention première était d'emprunter leurs indicateurs afin que les résultats de la présente recherche soient comparables aux leurs. Toutefois, un test préliminaire a mis en évidence la nécessité de développer des indicateurs collant mieux au caractère distinctif de cette recherche sur les PME de haute technologie. Cette démarche a débuté par l'identification des caractéristiques de la PME et des comportements de ses membres susceptibles de varier selon l'intensité de la veille. Des indicateurs ont ensuite été élaborés et des échelles bâties afin de traduire les données brutes en un score qui se prêterait mieux aux comparaisons. Une procédure similaire a été suivie pour toutes les autres variables observées.

L'intensité des activités de veille a été mesurée à l'aide des indicateurs et des mesures reproduits au tableau 1 qui suit, l'addition de ces mesures donnant un score global variant d'un minimum de 7 à un maximum de 33, représentant des activités très intenses.

Le concept de l'intégration de l'information au processus stratégique comporte, quant à lui, deux volets. Le premier a trait au processus par lequel l'information provenant de l'environnement est acheminée vers les membres de l'organisation, alors que la deuxième renvoie à l'usage subséquent de cette information à des fins stratégiques. Ce processus est susceptible de se distinguer selon la rapidité avec laquelle l'information circule, la fidélité de l'information transmise (Krone, Jablin et Putnam, 1987) et la pertinence de l'information qui y circule (Aguilar, 1967). L'usage de l'information recueillie à des fins stratégiques se vérifie en observant le mode de prise de décision du dirigeant et en tentant de décrire des situations concrètes où le dirigeant se souvient d'avoir utilisé des informations provenant des activités de veille. Le concept de l'intégration de l'information au 


\section{TABLEAU 1}

\section{Indicateurs et échelles de mesure pour l'intensité des activités de veille}

1) Proportion des membres de l'entreprise en position d'écoute.

$\begin{array}{lll}1=0 \text { à } 20 \% & 2=20 \text { à } 40 \% & 3=40 \text { à } 60 \% \\ 4=60 \text { à } 80 \% & 5=80 \% \text { et plus } & \end{array}$

2) Nombre de jours par année consacrés par des membres de l'entreprise à des congrès, conférences, activités de formation en rapport avec l'industrie ou foires commerciales. $1=0$ à $15 \quad 2=15$ à $30 \quad 3=30$ à 45 $4=45$ à $60 \quad 5=60$ et plus

3) Nombre mensuel de présence de membres de l'entreprise à des activités sociales d'affaires. $1=0$ à $2 \quad 2=2$ à $4 \quad 3=4$ à 6 $4=6$ à $8 \quad 5=8$ et plus

4) Proportion des membres consultant régulièrement les magazines spécialisés du domaine d'activités de l'entreprise.
$1=$ aucun ou quelques rares
$2=$ quelques-uns
$5=$ pratiquement tous
$3=$ près de la moitié
$4=$ la plupart

5) Proportion des membres naviguant régulièrement sur Internet.

$1=$ aucun ou quelques rares $\quad 2=$ quelques-uns $\quad 3=$ près de la moitié

$4=$ la plupart

$5=$ pratiquement tous

6) Fréquence moyenne des contacts du dirigeant avec les différents membres de son réseau d'information.

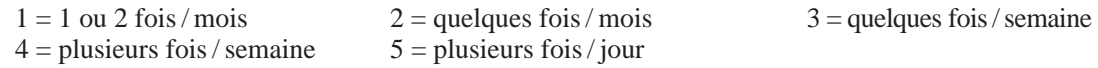

7) Nombre d'alliances stratégiques dans lesquelles l'entreprise est impliquée. $1=$ non impliquée $\quad 2=$ impliquée dans une alliance $\quad 3=$ impliquée dans plusieurs alliances

processus stratégique se traduit donc dans les mesures qui suivent au tableau 2, l'addition des deux dimensions du concept résultant en un score global du degré d'intégration de l'information allant d'un minimum de 6 à un maximum de 18.

Le concept d'adéquation entre l'intensité de la veille et l'incertitude stratégique perçue de l'environnement est une mesure d'association entre l'intensité de la veille et une combinaison du degré de turbulence, de complexité et d'importance des événements se produisant à l'intérieur d'un secteur donné $(\Downarrow$ Incertitude stratégique $=$ Importance $\%($ Complexité + Turbulence $) \downarrow ;$ Daft, Sormunen et Parks, 1988). Il a été demandé aux répondants de classer les différents secteurs de l'environnement selon leur importance, leur complexité et leur turbulence et selon le niveau des efforts de veille de l'ensemble des membres de l'entreprise. Pour ce faire, l'environnement externe a été subdivisé en sept secteurs distincts (clients, concurrents, fournisseurs, technologie, économie, gouvernement et secteur socioculturel). La mesure d'association retenue est le Rho de Spearman 
TABLEAU 2

Indicateurs et échelles de mesure pour l'intégration de l'information au processus stratégique

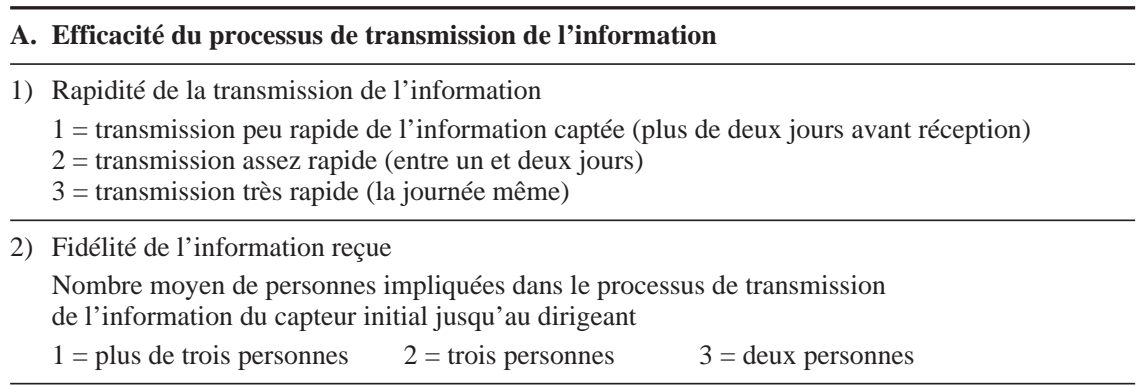

3) Pertinence de l'information recueillie et transmise

Évaluation du dirigeant quant au degré de pertinence pour lui des informations recueillies dans le cadre de la veille qui lui sont transmises par les membres de l'entreprise

1 = informations généralement peu pertinentes

$2=$ informations assez pertinentes

$3=$ informations très pertinentes

B. Usage stratégique de l'information recueillie lors de la veille

1) Importance de l'intuition par rapport à celle de l'information résultant de la veille dans la prise de décision

1 = rôle prépondérant de l'intuition

$2=$ partage plutôt égal entre les deux

3 = rôle prépondérant de l'information résultant de la veille

2) Considération accordée par le dirigeant aux opinions et informations transmises par d'autres personnes

$1=$ le dirigeant prend généralement ses décisions seul, sans consulter qui que ce soit.

$2=$ le dirigeant consulte à l'occasion.

$3=$ le dirigeant consulte régulièrement les personnes qui l'entourent et sollicite leur opinion.

3) Fréquence à laquelle le dirigeant est en mesure de relever des situations témoignant de l'usage de l'information recueillie

$1=$ le dirigeant peut difficilement relever plus d'une ou deux situations.

$2=$ le dirigeant est en mesure de relever quelques situations.

$3=$ le dirigeant peut facilement relever plusieurs situations.

puisque les variables étaient mesurées selon leur rang. Tout comme le $r$ de Pearson utilisé par Daft, Sormunen et Parks, ce coefficient oscille entre -1 et +1 , un coefficient se rapprochant de 1 indiquant que les valeurs élevées de la première variable (intensité de la veille) tendent à être pairées avec les valeurs élevées de la seconde variable (incertitude stratégique).

Finalement, pour mesurer le concept de performance, deux dimensions ont été retenues, soit celle de la rentabilité de l'entreprise (rendement sur les ventes) et celle de sa croissance (taux de croissance des ventes). Comme les informations 
comptables et financières des PME sont généralement de nature privée et, de surcroît, souvent peu fiables, la performance a été mesurée selon l'évaluation qu'en a fait le dirigeant en comparant son entreprise à ses principaux concurrents pour les trois dernières années financières. Soulignons que la mesure de performance n'était ici requise que pour distinguer, dans l'échantillon, les PME moins performantes des plus performantes, comme il sera expliqué plus loin.

Mentionnons que les variables exploratoires ont, elles aussi, été opérationnalisées. Une description des mesures et échelles qui ont été utilisées est reproduite en annexe.

\subsection{L'échantillon et la collecte des données}

L'échantillon est composé de quatre PME œuvrant dans des secteurs de moyenne à haute intensité technologique selon la classification proposée par l'Organisation du commerce et du développement économique. Plus précisément, les activités de ces PME se situent dans le domaine de la décontamination des sols par traitement biologique (Bioenv A et Bioenv B), dans la fabrication de pièces de haute précision à base de poudre de métal (Métal Inc.) et dans le secteur des technologies de l'information (Info Inc.). Elles comptent entre 18 et 120 employés et existent depuis au moins quatre ans et au plus onze ans. Elles sont toutes dirigées par une équipe d'entrepreneurs-fondateurs plutôt que par un seul propriétaire-dirigeant. Cette tendance semble d'ailleurs se confirmer (Gartner et al., 1994), surtout dans les milieux de la haute technologie.

La collecte des données s'est effectuée sous forme d'entrevues semi-dirigées avec les dirigeants et les employés les plus actifs en termes de veille au sein de l'entreprise. La durée de ces entrevues a varié entre deux et cinq heures par répondant et, en moyenne, deux répondants par entreprise ont été rencontrés. Dans une des quatre entreprises, un seul répondant a pu être rencontré. Des écrits de la presse spécialisée et des documents corporatifs sont alors venus compléter le témoignage du dirigeant pour assurer une bonne appréhension du contexte et du processus observés.

\subsection{L'analyse des données}

Comme nous l'avons dit, les observations recueillies ont d'abord fait l'objet d'analyses intrasites. Les données retenues à la suite de ce premier traitement ont alors été comparées et confrontées d'une entreprise à l'autre et de couples d'entreprises à d'autres, et ce afin de faire ressortir leurs similarités et différences. Pour ces fins, l'échantillon a été scindé selon le niveau de performance des entreprises, Bioenv A et Info Inc. étant les plus performantes et Bioenv B et Métal Inc., les moins performantes. L'objectif était de relever les caractéristiques de veille propres aux 
entreprises les plus performantes de l'échantillon. Plus précisément, ces caractéristiques devaient être communes aux deux PME les plus performantes et ne se retrouver chez aucune des entreprises moins performantes observées. Selon la logique sous-tendant ce type d'analyse, les caractéristiques de veille uniques aux PME les plus performantes observées sont vraisemblablement des caractéristiques associées à leur haut niveau de performance (d'Amboise, 1996). Ce sont ces caractéristiques de veille qui ont été retenues pour former le cadre conceptuel émergent présenté à la section suivante. Ces analyses par confrontations itératives ont par la suite été suivies d'une analyse complémentaire de type «élaboration d'explications ».

\section{Présentation et discussion des résultats obtenus}

Pour débuter, les aspects de la veille les plus intéressants qui ont été observés chez l'ensemble des PME seront décrits, accompagnés des scores obtenus pour les variables du cadre conceptuel et celles qualifiées d'exploratoires. Suivra ensuite la présentation du cadre conceptuel émergent, cadre qui comprend les dimensions de la veille qui se sont révélées uniques aux PME les plus performantes de l'échantillon.

\subsection{Description des activités de veille des PME de haute technologie}

Les pratiques de veille observées chez les PME de haute technologie ont certains points en commun. Tout d'abord, on remarque que l'intensité de leurs activités de veille est élevée (voir les pointages reproduits au tableau 3 ). Une forte proportion de leurs membres sont en position d'écoute (entre 50 et $100 \%$ d'entre eux), c'est-àdire qu'ils sont soit actifs en recherche et développement ou ont de fréquents contacts avec les fournisseurs, les clients, les concurrents ou d'autres intervenants de l'industrie. Les PME observées sont membres de plusieurs associations et participent à des tables de concertation ou grappes de développement reliées à leur industrie. De plus, elles sont partie à plusieurs alliances stratégiques. Cette forme de collaboration est d'ailleurs extrêmement importante pour ces PME puisqu'elle leur donne accès à de nouvelles technologies, de nouveaux marchés, des mandats lucratifs et contribuent à accélérer le développement de technologies et de procédés. À titre d'exemple, Bioenv A s'est liée de manière exclusive avec une grande entreprise canadienne pour pénétrer le marché américain. En vertu de cette entente, sa partenaire doit lui référer tous les mandats de traitement biotechnologique qu'elle obtiendra en sol américain et, en retour, Bioenv A s'est engagée à ne faire affaire que par son entremise aux États-Unis. Cette entente est fort avantageuse pour Bioenv A puisque le chiffre d'affaires de sa partenaire aux États-Unis dépasse déjà le milliard de dollars. Les alliances contribuent ainsi à la survie et à la croissance des PME observées et, de surcroît, elles constituent une 
excellente source d'informations de diverses natures. En rétrospective, comme toutes les PME de haute technologie observées ont des activités de veille très intenses, il semble que ce soit une condition nécessaire à leur survie sans que toutefois cela ne leur assure une performance supérieure.

Pour trois des quatre PME observées, l'information recueillie circule librement au sein de l'entreprise et se transmet rapidement et fidèlement jusqu'aux décideurs. Cette information est largement partagée entre les membres de l'entreprise, tant entre collègues qu'entre dirigeants et subalternes. Seule l'entreprise Bioenv $\mathrm{B}$ éprouve des problèmes de communication interne. Fait intéressant, lors de l'entrevue, le dirigeant de Bioenv $\mathrm{B}$ a relevé ce problème et a mentionné que des mesures avaient été prises pour y remédier.

Le style de prise de décision favorisé par les dirigeants des PME de haute technologie est à la fois collectif et consensuel. Ce style laisse place aux données factuelles recueillies dans le cadre de la veille : les dirigeants pouvaient facilement se rappeler de situations où leur décision avait été influencée par des informations provenant des activités de veille.

TABLEAU 3

\section{Scores relatifs aux variables du cadre conceptuel}

Métal Inc. Info Inc. Bioenv A Bioenv B

Intensité des activités de veille

Pourcentage d'employés en position d'écoute / 5

Participation à des activités externes / 10

Lectures spécialisées et navigation Internet / 10

Fréquence des contacts avec réseau d'information / 5

Participation à des alliances stratégiques / 3

Total / 33

Intégration de l'information au processus stratégique

Efficacité de la transmission / 9

Usage stratégique de l'information recueillie / 9

Total / 18

\begin{tabular}{rrrr}
3 & 5 & 4 & 4 \\
5 & 5 & 8 & 8 \\
7 & 10 & 4 & 4 \\
5 & 5 & 5 & 3 \\
3 & 3 & 3 & 3 \\
23 & 28 & 24 & 22 \\
\hline
\end{tabular}

Adéquation entre l'intensité de la veille

et l'incertitude stratégique

\begin{tabular}{lllll} 
Rho de Spearman & 0,71 & 0,76 & 0,68 & 0,42 \\
\hline Performance /8 & 5 & 6 & 6,5 & 4,5 \\
\hline
\end{tabular}

Pour trois des PME observées, l'intensité des activités de veille variait en fonction du niveau d'incertitude stratégique que leurs dirigeants percevaient dans l'environnement (voir tableau 4), Bioenv B étant la seule exception. C'est donc dire que les efforts de veille étaient prioritairement dirigés vers les secteurs de 
TABLEAU 4

\section{Rangs attribués par le dirigeant aux secteurs de l'environnement} (du $1^{\mathrm{er}}$ au $7^{\mathrm{e}}$ rang)

\begin{tabular}{|c|c|c|c|c|c|c|c|}
\hline & $\begin{array}{c}\text { Client } \\
\text { et marché }\end{array}$ & Technologie & Concurrents & Fournisseurs & Économie & Gouvernement & $\begin{array}{l}\text { Société } \\
\text { et culture }\end{array}$ \\
\hline \multicolumn{8}{|l|}{ Métal Inc. } \\
\hline Importance du secteur & 2 & 5 & 4 & 3 & 6 & 1 & 7 \\
\hline Turbulence perçue & 1 & 4 & 5 & 3 & 6 & 2 & 7 \\
\hline Complexité perçue & 4 & 2 & 1 & 5 & 6 & 3 & 7 \\
\hline \multicolumn{8}{|l|}{ Incertitude stratégique } \\
\hline$(\mathrm{I}[\mathrm{T}+\mathrm{C}])$ & 2 & 5 & 3,5 & 3,5 & 6 & 1 & 7 \\
\hline Intensité de la veille & 1 & 2 & 5 & 4 & 6 & 3 & 7 \\
\hline \multicolumn{8}{|l|}{ Rho de Spearman : 0,71 } \\
\hline \multicolumn{8}{|l|}{ Info Inc. } \\
\hline Importance du secteur & 1 & 4 & 2 & 6 & 5 & 3 & 7 \\
\hline Turbulence perçue & 1 & 3 & 2 & 6 & 5 & 4 & 7 \\
\hline Complexité perçue & 1 & 3 & 2 & 6 & 5 & 4 & 7 \\
\hline \multicolumn{8}{|l|}{ Incertitude stratégique } \\
\hline$(\mathrm{I}[\mathrm{T}+\mathrm{C}])$ & 1 & 3,5 & 2 & 6 & 5 & 3,5 & 7 \\
\hline Intensité de la veille & 2 & 1 & 4 & 5 & 6 & 3 & 7 \\
\hline \multicolumn{8}{|l|}{ Rho de Spearman : 0,76 } \\
\hline \multicolumn{8}{|l|}{$\overline{\text { Bioenv A }}$} \\
\hline Importance du secteur & 4 & 2 & 3 & 5 & 6 & 1 & 7 \\
\hline Turbulence perçue & 2 & 4 & 1 & 5 & 6 & 3 & 7 \\
\hline Complexité perçue & 2 & 1 & 3 & 5 & 6 & 4 & 7 \\
\hline \multicolumn{8}{|l|}{ Incertitude stratégique } \\
\hline$(\mathrm{I}[\mathrm{T}+\mathrm{C}])$ & 4 & 2 & 3 & 5 & 6 & 1 & 7 \\
\hline Intensité de la veille & 1 & 2 & 3 & 5 & 6 & 4 & 7 \\
\hline \multicolumn{8}{|l|}{ Rho de Spearman : 0,68 } \\
\hline \multicolumn{8}{|l|}{ Bioenv B } \\
\hline Importance du secteur & 1 & 4 & 2 & 5 & 7 & 3 & 6 \\
\hline Turbulence perçue & 1 & 6 & 2 & 7 & 5 & 4 & 3 \\
\hline Complexité perçue & 2 & 5 & 3 & 6 & 7 & 4 & 6 \\
\hline \multicolumn{8}{|l|}{ Incertitude stratégique } \\
\hline$(\mathrm{I}[\mathrm{T}+\mathrm{C}])$ & 1 & 5 & 2 & 6 & 7 & 3,5 & 3,5 \\
\hline Intensité de la veille & 1 & 2 & 4 & 3 & 7 & 5 & 6 \\
\hline Rho de Spearman : 0,42 & & & & & & & \\
\hline
\end{tabular}

l'environnement perçus comme les plus importants, complexes et turbulents. À noter toutefois que les dirigeants des quatre PME technologiques observées ont attribué un rang peu élevé au secteur de la technologie en termes de turbulence alors que ce secteur était scruté avec beaucoup d'intensité. Il est plutôt étonnant que la technologie ne soit pas perçue comme un secteur de l'environnement d'affaires suscitant beaucoup de turbulence. L'explication se dégageant des entrevues est que, d'une part, les dirigeants ont l'impression de bien maîtriser leurs technologies et que, d'autre part, ces technologies évoluent de manière incrémentale, voire prévisible. 
Des pratiques de veille intéressantes ont aussi été relevées du côté des variables exploratoires (voir le tableau 5 pour les pointages).

\section{TABLEAU 5}

\section{Scores relatifs aux variables exploratoires}

\begin{tabular}{lcccc}
\hline & Métal Inc. Info Inc. Bioenv A Bioenv B \\
\hline Niveau de structuration des activités de veille / & 3 & 2,5 & 3 & 2 \\
Horizon temporel de la veille / 3 (3 = long terme) & 3 & 3 & 3 & 2,5 \\
Motivation sous-tendant la veille / 3 (3= prospective) & 3 & 3 & 3 & 2,5 \\
Place de la veille dans la culture / 3 (3= essentielle) & 3 & 3 & 3 & 2 \\
Efficacité du réseau d'information & & & \\
$\quad$ Diversité du réseau / 3 & 2 & 2 & 2 & 2 \\
Niveau de confiance / 3 & 3 & 3 & 3 & 2 \\
$\quad$ Résultats des efforts / 5 & 3 & 5 & 5 & 3 \\
Total / 11 & 8 & 10 & 10 & 7 \\
\hline
\end{tabular}

Pour trois des PME observées, les activités de veille étaient bien structurées. Plus précisément, des responsabilités de veille avaient été assignées à des membres de l'entreprise, ces derniers avaient des objectifs de surveillance et de collecte, et ils suivaient une méthodologie pour les réaliser. À titre illustratif, chez Bioenv A, les fonctions de la directrice de la microbiologie appliquée incluent la pratique d'une veille technologique anticipative. À chaque mois, elle va à la bibliothèque universitaire consulter une vingtaine de périodiques scientifiques qu'elle a préalablement sélectionnés. Elle prend également connaissance des articles parus dans une cinquantaine d'autres revues spécialisées. Son processus de recherche d'informations est structuré, d'une part, autour de mots clés et, d'autre part, autour d'objectifs de surveillance. La directrice cherche notamment à savoir quels sont les concurrents actifs sur un site de décontamination et quels sont leurs procédés.

Les activités de veille sont généralement valorisées au sein des PME observées, les encouragements étant probablement plus sentis au sein des équipes de recherche. Il n'y a que chez Bioenv B que la veille n'est pas prisée : les membres de cette organisation ne sont pas récompensés pour leurs efforts de veille et, de façon générale, ils n'ont pas la mentalité du partage de l'information.

Fait assez remarquable, chez toutes les PME, il existe une multiplicité de réseaux d'information. Il n'appartient donc pas uniquement au chef de l'entreprise de se bâtir un réseau de contacts ; plusieurs membres de l'entreprise contribuent aux efforts de veille de cette façon, ayant développé leur propre réseau de contacts au cours des années. L'entreprise jouit donc de réseaux couvrant un large spectre d'acteurs, que ce soit de la communauté scientifique, de l'industrie ou simplement du milieu des affaires.

Revue internationale P.M.E., vol. 16, $\mathrm{n}^{\circ}$ 2, 2003 


\subsection{Le cadre conceptuel émergent}

Les analyses intersites effectuées avec les données recueillies auprès des PME de haute technologie ont permis de dégager un cadre conceptuel émergent (voir la figure 2).

FIGURE 2

Cadre conceptuel émergent des activités de veille des PME de haute technologie

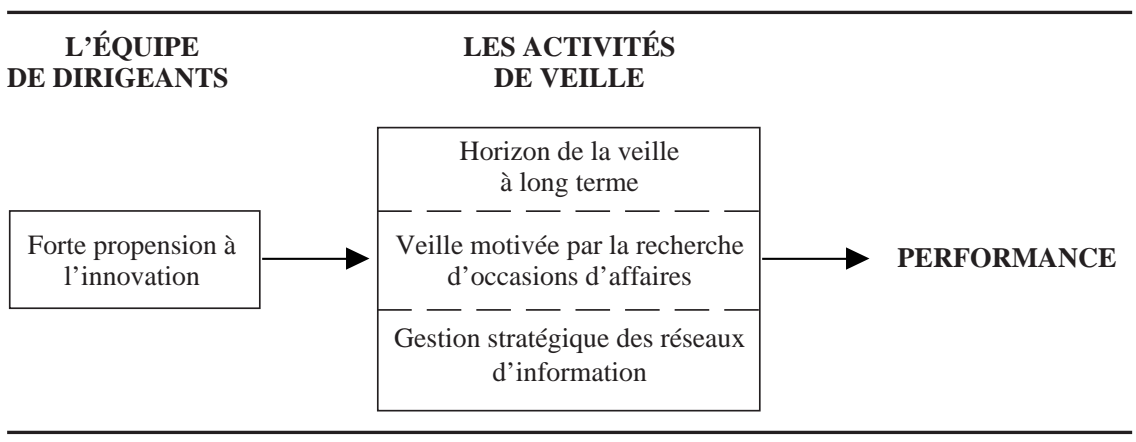

Les analyses ont fait ressortir chez les PME plus performantes une variable qui, sans être une dimension de la veille, a néanmoins un impact sur elle : la propension à l'innovation de l'équipe dirigeante. Ce désir d'innover, de faire les choses différemment, sert de catalyseur à la veille puisqu'il amplifie les besoins informationnels. Ainsi, ce qui distingue Bioenv A de ses concurrents, c'est sa capacité à développer des procédés de décontamination innovateurs, année après année. Cette PME a choisi de s'attaquer aux problématiques les plus complexes, mettant au point des procédés pour traiter de manière efficace et économique une large gamme de contaminants habituellement réfractaires à la biodégradation. Aux premières heures de l'entreprise, les deux dirigeants ont fait plusieurs choix technologiques très audacieux, osant aller de l'avant avec des procédés qui n'avaient jamais été essayés avec le type de contaminants qu'ils avaient à traiter. Ils ont ainsi gagné le respect et l'admiration de leurs employés qui les considèrent comme des visionnaires « high tech», des patrons qui ont du cran. Encore aujourd'hui, les dirigeants encouragent fortement les employés à chercher de nouvelles idées, à innover, ce qui stimule leur recherche d'informations de toutes sortes.

Chez Info Inc., l'innovation n'est pas tant technologique que stratégique; cette PME se démarque de la concurrence en misant à la fois sur son expertise technologique et sur une connaissance en profondeur du secteur de la santé dans lequel évoluent ses clients. Leur force repose sur leur capacité à aller chercher l'information et à l'intégrer dans leurs produits. Les dirigeants ne ménagent aucun 
effort pour que l'entreprise soit à la fine pointe de la technologie, comme en fait foi leur décision de donner à tous les employés un accès direct et illimité à l'«autoroute électronique » dès 1992. Ils sont avides d'informations autant en ce qui a trait au fonctionnement du système de santé québécois qu'aux besoins de leur clientèle.

Les caractéristiques de la veille qui se sont révélées uniques aux PME les plus performantes sont l'horizon à long terme de cette veille, sa concentration sur la recherche d'occasions d'affaires et une gestion stratégique des réseaux d'information des membres de l'entreprise. Ainsi, l'horizon temporel des activités de veille est plus orienté vers le long terme que celui des PME moins performantes. Leur veille est plutôt prospective, la motivation dominante étant de déceler les occasions d'affaires avant quiconque de manière à les exploiter et ainsi être des précurseurs. Cela contraste avec les PME moins performantes qui, elles, se heurtent souvent à des problèmes d'ordre technique les obligeant à concentrer leurs efforts de veille sur le court terme afin de trouver rapidement des solutions.

Comme nous l'avons déjà mentionné, l'ensemble des PME observées jouissaient de réseaux de contacts bien établis. Ce qui distingue les PME performantes des moins performantes, c'est le soin et l'attention portés à la constitution et à la gestion de ces réseaux. Ainsi, chez les moins performantes, le partage des responsabilités de veille parmi les différents réseauteurs s'est fait un peu par la force des choses alors que chez les plus performantes, il a été soigneusement planifié et orchestré. Plus précisément, les dirigeants ont préalablement repéré les groupes d'intervenants ou d'acteurs auprès desquels il serait important que l'entreprise établisse des contacts. Dans un deuxième temps, les membres de l'entreprise ayant le plus d'affinités avec les groupes ciblés se sont vu déléguer la responsabilité d'y développer un réseau de contacts, avec instructions de partager avec les autres membres de l'entreprise toutes les informations pertinentes qu'ils y recueilleraient.

Mentionnons que les dirigeants des PME plus performantes sont particulièrement inventifs lorsqu'il s'agit de trouver une information par l'entremise de leurs réseaux. À titre illustratif, dans le cadre d'un projet pour lequel Info Inc. travaillait en collaboration avec un concurrent, le dirigeant s'est trouvé aux prises avec un problème d'ordre administratif qui ralentissait l'exécution du projet. Il a alors convaincu son partenaire-concurrent de dresser un tableau décrivant la toile d'araignée que formaient leurs réseaux de contacts respectifs, et ce dans le but de découvrir lequel de leurs contacts était le mieux placé pour les aider à faire avancer le projet.

L'explication élaborée à la suite des analyses par comparaisons pour donner un sens aux différentes variables et relations composant le cadre émergent est la suivante. Les dirigeants des PME de haute technologie les plus performantes de l'échantillon sont des visionnaires animés par un fort désir d'innover, d'être des

Revue internationale P.M.E., vol. 16, $\mathrm{n}^{\circ}$ 2, 2003

(C) 2003 - Presses de l'Université du Québec 
précurseurs. Ce désir stimule les activités de veille tout en leur donnant l'orientation nécessaire pour qu'il soit satisfait. La motivation qui dirige la veille est donc une volonté ferme de précéder les tendances et de détecter rapidement les occasions d'affaires, les informations requises pour arriver à ces fins relevant principalement d'un horizon à long terme. Par ailleurs, ces dirigeants font une gestion stratégique des différents réseaux d'information auxquels les membres de l'entreprise sont reliés. L'importance accordée par l'équipe de dirigeants à la constitution de ces réseaux est liée à ce profond besoin d'innover puisque c'est, entre autres, à travers ces réseaux que l'entreprise obtient les informations requises pour se positionner à l'avant-garde de l'industrie.

\section{Conclusion}

\section{Regard orienté vers l'avenir}

Comme la relation existant entre la veille stratégique et la performance de l'entreprise était un phénomène peu documenté et encore mal compris, l'objectif premier de cette recherche était d'ébaucher une première tentative d'explication du phénomène de la veille chez les PME de haute technologie. Non seulement cet objectif a été atteint, mais les résultats ont aussi mis en évidence des caractéristiques du processus de veille dont la contribution au succès de l'entreprise était jusque-là insoupçonnée. De fait, les variables constituant le cadre émergent sont toutes des dimensions de la veille dont l'association à la performance de la PME n'avait pas encore été démontrée. Ainsi, le rôle prépondérant du dirigeant de PME dans le processus de veille a été mis en lumière, son désir d'innover servant de bougie d'allumage aux activités de veille, stimulant et orientant les efforts de veille de l'ensemble des membres de l'entreprise. Par ailleurs, bien que certains auteurs aient déjà découvert l'existence d'un lien entre les activités de réseautage et la croissance de la PME (Hansen, 1995 ; Ostgaard et Birley, 1996), les particularités de la constitution des réseaux de contacts au sein de la PME n'avaient pas été prises en compte de façon spécifique. Or, les résultats indiquent qu'une des caractéristiques qui distinguent les PME les plus performantes de l'échantillon est l'attention et l'ingéniosité avec lesquelles les réseaux de contacts ont été planifiés et mis en place.

Il est à espérer que l'intérêt suscité par les résultats obtenus encourage la poursuite d'autres recherches ayant pour but de les vérifier et, le cas échéant, de les rendre généralisables à un plus grand nombre de PME. En effet, comme les fruits de la présente recherche proviennent de l'analyse des données recueillies auprès de seulement quatre PME de haute technologie, cette étude est largement exploratoire, de nature à suggérer des liens potentiels plutôt qu'à confirmer des liens réels. Une dernière mise en garde s'impose : comme la collecte des données n'a pas été faite de manière longitudinale mais a plutôt eu lieu à un seul point dans 
le temps, il n'a pas été possible de vérifier s'il y avait un décalage temporel entre l'adoption de pratiques de veille particulières et l'effet de telles pratiques sur la performance de l'entreprise.

\section{ANNEXE I \\ Indicateurs et échelles de mesure pour les variables exploratoires}

- Degré de structuration des activités de veille

1) Existence de membres assignés à des activités de veille

$1=$ oui $\quad 2=$ non

2) Existence d'objectifs de surveillance et de collecte d'informations

$1=$ oui $\quad 2=$ non

3) Existence d'une méthodologie particulière pour surveiller l'environnement

et/ou recueillir les informations

Score maximal : 3 activités de veille très structurées

Score minimal : $0 \quad$ absence de structuration des activités de veille

\section{- Horizon temporel des activités de veille}

Score de 1: La plupart des informations recueillies relèvent d'un horizon temporel à très court terme ; ce sont généralement des informations de nature courante pour usage immédiat.

Score de 2: La plupart des informations recueillies relèvent d'un horizon temporel à court terme ; ce sont généralement des informations concernant des événements ou situations susceptibles de se produire dans le courant de l'année.

Score de 3: La plupart des informations recueillies relèvent d'un horizon temporel à long terme ; ce sont généralement des informations concernant des événements ou situations susceptibles de se produire au-delà de l'année courante.

- Motivation sous-tendant les efforts de veille

Score de 1: La collecte de la plupart des informations de veille est déclenchée par des crises au sein de l'entreprise.

Score de 2: La collecte de la plupart des informations de veille est motivée par la recherche d'une solution à un problème dans l'entreprise.

Score de 3: La collecte de la plupart des informations de veille est motivée par la recherche d'occasions d'affaires pour l'entreprise ou a pour but de détecter les menaces éventuelles à la pérennité de l'entreprise.

- Place de la veille au sein de la culture organisationnelle

Score de 1: Les activités de veille ne sont pas intégrées aux activités de base de l'entreprise. Le dirigeant ne valorise pas du tout la veille et n'encourage pas les membres à surveiller l'environnement ou à recueillir des informations. Les membres de l'organisation ne se sentent pas encouragés à faire de la veille et n'ont pas l'impression qu'il s'agit d'une activité qui a de la valeur pour l'entreprise. Ils ne croient pas que des efforts de veille seraient appréciés ou récompensés.

Score de 2: Les activités de veille sont marginales par rapport aux activités de base de l'entreprise. Le dirigeant est plus ou moins convaincu de la nécessité de faire de la veille ou de ses bienfaits éventuels. Bien que pratiquant lui-même une certaine forme de veille, il n'encourage pas ouvertement les membres à surveiller l'environnement ou à recueillir des informations. Les employés sont peu ou pas conscients de la nécessité de pratiquer une veille et doutent que de tels efforts seraient appréciés ou récompensés.

Revue internationale P.M.E., vol. 16, nº 2, 2003 
Score de 3: Les activités de veille sont considérées essentielles à la survie à long terme de l'entreprise.

Le dirigeant est convaincu de la nécessité de faire de la veille et il encourage ouvertement les membres de l'entreprise à surveiller l'environnement ou à recueillir des informations. Les employés sont conscients de l'importance accordée aux activités de veille et savent que leurs efforts seront appréciés.

- Efficacité du réseau d'information du dirigeant à générer des informations riches

Diversité du réseau

Nombre de catégories distinctes représentées par les individus formant le réseau d'information du dirigeant

Catégories possibles (7):

- fournisseur - sous-traitant - concurrent - autre

- client - professionnel - centre de recherche ou organisme gouvernemental

Score de 1: Réseau peu diversifié (1 ou 2 catégories représentées)

Score de 2: Réseau assez diversifié (3 ou 4 catégories représentées)

Score de 3: Réseau très diversifié (plus de 4 catégories représentées)

Confiance régnant au sein du réseau

Score de 1: Degré de confiance plus ou moins élevé dans la majorité des membres du réseau

Score de 2: Degré de confiance assez élevé dans la majorité des membres du réseau

Score de 3: Degré de confiance très élevé dans la majorité des membres du réseau

Résultats des efforts de veille par l'entremise du réseau

Score de 1: Le dirigeant ne recueille jamais ou presque des informations riches auprès des membres de son réseau.

Score de 2: Le dirigeant recueille à l'occasion des informations riches auprès des membres de son réseau.

Score de 3: Le dirigeant recueille assez souvent des informations riches auprès des membres de son réseau.

Score de 4: Le dirigeant recueille souvent des informations riches auprès des membres de son réseau.

Score de 5: Le dirigeant recueille très souvent des informations riches auprès des membres de son réseau.

Mesure de l'efficacité du réseau à générer

des informations riches $=$ total des scores obtenus

Score maximal : $11 \quad$ Réseau très efficace à générer des informations riches.

Score minimal : $\quad 3$ Réseau très peu efficace à générer des informations riches.

\section{Bibliographie}

Aguilar, F.J. (1967), Scanning the Business Environment, New York, Macmillan.

AUSTER, E. et C.W. CHOO (1994), «How senior managers acquire and use information in environmental scanning », Information Processing \& Management, vol. 30, $\mathrm{n}^{\circ} 5$, p. 607-618.

BEAL, R.M. (2000), «Competing effectively: environmental scanning, competitive strategy, and organizational performance in small manufacturing firms », Journal of Small Business Management, vol. 38, n 1, p. 27-47.

Revue internationale P.M.E., vol. 16, $n^{\circ}$ 2, 2003 
BoYD, B.K. (1989), Perceived Uncertainty and Environmental Scanning : A Structural Model, Thèse de Ph. D., University of Southern California.

CHOo, C.W. (1993), Environmental Scanning: Acquisition and Use of Information by Chief Executive Officers in the Canadian Telecommunication Industry, Thèse de Ph.D., Université de Toronto.

Cooper, A.C., T.B. Folta et C. Woo (1995), «Entrepreneurial information search», Journal of Business Venturing, vol. 10, p. 107-120.

COURVILLE, L. (1994), Piloter dans la tempête : comment faire face aux défis de la nouvelle économie?, Montréal, Québec/Amérique.

DAFT, R.L., J. SORMUNEN et D. PARKS (1988), «Chief executive scanning, environmental characteristics, and company performance : an empirical study », Strategic Management Journal, vol. 9, p. 123-139.

D’AMBoise, G. (1996), Le projet de recherche en administration : Un guide général à sa préparation, disponible sur Internet <http:// www.fsa.ulaval.ca/personnel/damboisg/ liv1>. [Accès le 4 janvier 2001.]

Dess, G. et A. MiLler (1993), Strategic Management, New York, McGraw-Hill.

DOLLINGER, M.J. (1984), «Environmental boundary spanning and information processing effects on organizational performance», Academy of Management Journal, vol. $27, \mathrm{n}^{\circ} 2$, p. $351-368$.

DONCKELS, R. et J. LAMBRECHT (1997), «The network position of small businesses : an explanatory model », Journal of Small Business Management, vol. 35, $\mathrm{n}^{\circ}$ 2, p. 13-25.

DUBINI, P. et H. ALDRICH (1991), «Personal and extended networks are central to the entrepreneurial process », Journal of Business Venturing, vol. 6, p. 305-313.

EISENHARDT, K.M. (1989), «Building theories from case study research », Academy of Management Review, vol. 14, no 4, p. 532-550.

FAHEY, L. et W.R. KING (1977), «Environmental scanning for corporate planning», Business Horizons, août, p. 61-71.

Gartner, W.B., K.G. Shaver, E. Gatewood et J.A. Katz (1994), «Finding the entrepreneur in entrepreneurship », Entrepreneurship, Theory and Practice, vol. 18, $\mathrm{n}^{\mathrm{o}} 3$, p. 5-10.

HAGEN, A.F. et S.G. AMIN (1995), «Corporate executives and environmental scanning activities: an empirical investigation », SAM Advanced Management Journal, printemps, p. 41-47.

HAMBRICK, D.C. (1981), « Specialization of environmental scanning activities among upper level executives », Journal of Management Studies, vol. 18, nº 3, p. 299-320.

HAMBRICK, D.C. (1982), «Environmental scanning and organizational strategy », Strategic Management Journal, vol. 3, p. 159-174.

HAMEl, G. et C.K. Prahalad (1994), Competing for the Future, Boston, Harvard School Press.

HANSEN, E.L. (1995), «Entrepreneurial network and new organization growth», Entrepreneurship, Theory and Practice, vol. 19, no 4, p. 7-19.

Revue internationale P.M.E., vol. 16, nº 2, 2003

(C) 2003 - Presses de l'Université du Québec 
Huberman, M.A. et M.B. Miles (1991), Analyse des données qualitatives : Recueil de nouvelles méthodes, Bruxelles, DeBoeck-Wesmael.

JAIN, S.C. (1984), «Environmental scanning in U.S. corporations », Long Range Planning, vol. 17, n 2, p. 117-128.

JENNINGS, D.F. et J.R. LUMPKIN (1992), «Insights between environmental scanning activities and Porter's generic strategies: an empirical analysis », Journal of Management, vol. 18, $\mathrm{n}^{\circ}$ 4, p. 791-803.

JoHnson, J.L. et R. KUEHN (1987), «The small business owner/manager's search for external information», Journal of Small Business Management, vol. 25, $\mathrm{n}^{\circ} 3$, p. 53-60.

JULIEN, P.-A. (1996), « Information control : a key factor in small business development », Communication présentée au 41st ICSB World Conference, Stockholm, 17-19 juin.

Julien, P.-A., L. RAYMOND, R. JACOB et C. RAMANGALAhY (1999), «Types of technological scanning in manufacturing SMEs : an empirical analysis of patterns and determinants», Entrepreneurship and Regional Development, vol. 11, p. 281-300.

KAISH, S. et B. GILAD (1991), «Characteristics of opportunities search of entrepreneurs versus executives: sources, interests, general alertness », Journal of Business Venturing, vol. 6, p. 45-62.

Krone, B., F.M. Jablin et L.L. Putnam (1987), «Communication theory and organizational communication: multiple perspectives», dans F.M Jablin, L.L. Putnam, K.H. Roberts et L.W. Porter (dir.), Handbook of Organizational Communication, Newbury Park, Sage Publications, p. 18-40.

LESCA, H. (1994), «Veille stratégique pour le management stratégique : État de la question et axes de recherche», Économies et Sociétés, vol. 20, nº 5, p. 31-50.

LESCA, H. et M.-L. CARON (1995), «Veille stratégique : créer une intelligence collective au sein de l'entreprise », Revue française de gestion, septembre-octobre, p. 58-68.

MARTINET, B. et J.M. RIBAULT (1989), La veille technologique, concurrentielle et commerciale. Sources, méthodologie, organisation, Paris, Les Éditions d'Organisations.

Merriam, S.B. (1998), Qualitative Research and Case Study Applications in Education, San Francisco, Jossey-Bass.

OSTGAARD, A. et S. BIRLEY (1996), «New venture growth and personal networks », Journal of Business Research, vol. 36, p. 37-50.

OugheBBI, A. (1993), «Le processus d'acquisition de l'information stratégique dans les PME : le dirigeant en tant que pôle de décision pertinent », dans M.-P. Bes et J.-L. Leboulch (dir.), L'information face au changement technique, Paris, L'Harmattan, p. 351-368.

PAteyron, E. (1998), La veille stratégique, Paris, Economica.

PeArCE II, J.A., B.L. Chapman et F.R. DAVID (1982), «Environmental scanning for small and growing firms », Journal of Small Business Management, vol. 20, $\mathrm{n}^{\circ} 3$, p. 27-35.

Revue internationale P.M.E., vol. 16, $n^{\circ}$ 2, 2003 
PinedA, R.C., L.D. LERnER, M.C. MiLler et S.J. PhILliPS (1998), «An investigation of factors affecting the information-search activities of small business managers », Journal of Small Business Management, vol. 36, n 1, p.60-71.

SAWYERR, O.O. (1993), «Environmental uncertainty and environmental scanning activities of Nigerian manufacturing executives : a comparative analysis », Strategic Management Journal, vol. 14, p. 287-299.

SAWYERR, O.O. et J.E. MCGEE (1999), « The impact of personal network characteristics on perceived environmental uncertainty: an examination of owners/managers of new high technology firms », Frontiers of Entrepreneuship Research, Babson College.

SMELTZER, L.R., G.L. FANN et N. NIKOLAISEN (1988), «Environmental scanning practices in small business », Journal of Small Business Management, vol. 26, $\mathrm{n}^{\circ}$ 3, p. 55-62.

Subramanian, R., K. Kumar et C. YAUger (1994), « The scanning of task environments in hospitals : an empirical study », Journal of Applied Business Research, vol. 10, $\mathrm{n}^{\circ} 4$, p. 104-115.

YIN, R.K. (1984), Case Study Research: Design and Methods, Beverly Hills, Cal., Sage Publications.

YIN, R.K. (1993), Applications of Case Study Research, Newbury Park, Sage Publications.

YIN, R.K. (1994), Case Study Research : Design and Methods, $2^{\mathrm{e}}$ édition, Thousand Oaks, Cal., Sage Publications.

Revue internationale P.M.E., vol. 16, $\mathrm{n}^{\circ}$ 2, 2003 